\section{¿La movilidad de las personas mayores es sustentable? El caso de la Ciudad de Buenos Aires, Argentina}

Mariana Cataldi

Carrera de Trabajo Social, Facultad de Ciencias Sociales, Universidad de Buenos Aires / Carrera de Trabajo Social, Universidad Nacional de José C. Paz, Argentina https://orcid.org/oooo-0003-1553-8535

Recibido: 8 de abril de 2018. Aceptado: 28 de junio de 2019.

\title{
Resumen
}

El trabajo describe las prácticas de movilidad de las personas mayores en la Ciudad de Buenos Aires, Argentina, y analiza sus dimensiones con relación a la sustentabilidad. A partir de una muestra conformada por 40 personas mayores de 60 años que asisten a servicios de salud, se describen sus movilidades, los modos de uso y no uso de transporte, los significados de sus prácticas y se analiza esta información en el marco del nuevo paradigma de movilidad sustentable.

\section{Is the mobility sustainable? The case of the City of Buenos Aires, Argentina}

\begin{abstract}
The work describes the mobility practices of the elderly in the City of Buenos Aires, Argentina, and analyzes their dimensions in relation to sustainability. Based on a sample of 40 people over 60 years of age who attend health services, their mobilities, modes of use and non-use of transport, the meanings of their practices are described and this information is analyzed within the framework of the new paradigm of sustainable mobility.
\end{abstract}

\section{Introducción}

El mundo está envejeciendo. En los últimos años, la población de mayor edad ha registrado un incremento sostenido y se estima que en el año 2050 el 22\% de habitantes tendrá 60 años y más. En 2030, el número de personas mayores superará al de niños y se predice que en 2050 habrá más personas mayores que adolescentes y jóvenes (OMS,

\section{Palabras clave}

Movilidad Transporte Sustentabilidad Personas mayores

Palavras-chave

Mobilidade Transporte Sustentabilidade Pessoas maiores
Keywords

Mobility Transport Sustainability
Old people

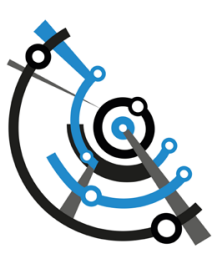


2007). Por lo general, las grandes ciudades presentan altos índices de población mayor pero no han sido diseñadas ni adecuadas a sus necesidades e intereses particulares.

De acuerdo a datos provenientes del último Censo Nacional realizado en el año 2010, en la Ciudad de Buenos Aires (en adelante CABA) residen 2.890.151 personas (INDEC, 2010), por lo cual se ubica dentro de las ciudades denominadas "millonarias" (Da Cunha, 2009). A la población residente permanente se agregan los transitorios, como trabajadores, turistas y otros visitantes ocasionales. Diariamente 3,2 millones de residentes del conurbano ingresan a la Ciudad para trabajar (DGT, 2010). Todos ellos circulando en el mismo espacio. Esta concentración ha derivado en múltiples problemas urbanos, como la insuficiencia de servicios públicos o la inadecuación de los mismos a las necesidades de la población que allí reside, entre ellos el transporte.

El 16,4 \% de los habitantes de la CABA son hombres y mujeres mayores de 65 años y la cantidad de población femenina supera a la masculina. El 5,1\% son mayores de 80 años. Según Naciones Unidas, se considera una población "envejecida" cuando supera el 7\% de mayores. Es "realmente envejecida" cuando es superior a 12,5\% (Laslett, 1989). La CABA se encuentra en esta última categoría. Junto a Santa Fe, La Pampa y Córdoba integra el grupo de ciudades más añosas de Argentina. El envejecimiento demográfico urbano, ha sido considerado como "un hecho sin precedentes y problemático" (Sánchez González, 2015) que ha dado lugar a numerosos interrogantes sobre los problemas a que se enfrentan las personas mayores en su entorno: alta concentración urbana y dispersión rural, diferencias en la calidad de vida, desigualdades.

En el Área Metropolitana de Buenos Aires, en los años 2009-2010 el Ministerio de Transporte aplica la Encuesta de Movilidad Domiciliaria (ENMODO) que pretende conocer los patrones de movilidad. Los resultados arrojan algunos de los principales hallazgos sobre la movilidad de jubilados y pensionados, grupo poblacional definido en ese informe por su actividad y no por su edad cronológica. Observan bajos niveles de viajes, tanto en hombres como en mujeres y priman los viajes de corta duración, el $61 \%$ responde que no viajó el día anterior. Sobre la elección del modo, es predominante el transporte público y, dentro del mismo, el $80 \%$ utiliza colectivo, siendo superior la elección de este modo en las mujeres (UEC, 2010). En producciones específicas sobre las condiciones de vida de las personas mayores también se señalan algunas particularidades sobre la movilidad de las personas mayores. Casi la mitad de las de 75 años y más presentan algún tipo de dependencia instrumental, en la que se incluye la necesidad de apoyo en el transporte (INDEC, 2012). La inaccesibilidad en el transporte es considerada una barrera urbanística dado que constituye un obstáculo para la movilidad de las personas mayores en el espacio urbano (MDS, 2010). La movilidad de las personas mayores fue analizada desde distintas perspectivas, como la económicopolítica, social, ambiental-urbanística, el enfoque de derechos. Entre los principales hallazgos se menciona la estrecha relación entre el factor económico y la movilidad, dado que ésta aumenta a medida que aumenta el ingreso. Las personas mayores de menores recursos utilizan principalmente modos de transporte público colectivo, se trasladan a pie u omiten viajar (Cataldi, 2018).

El trabajo parte de las siguientes premisas: la movilidad es un derecho, un derecho que permite el acceso a otros. Es un concepto más amplio que el de transporte, ya que considera los modos de traslado motorizados y no motorizados, individuales y colectivos, públicos y privados e incorpora los significados de dichas prácticas. La perspectiva de la movilidad permite definir las prácticas sociales de viaje, motivadas por un deseo o necesidad y para el logro de una finalidad. Este enfoque propone delimitar el caso o modelo a partir de un grupo social tipo; personas mayores, un territorio tipo; CABA, y un fin de viaje; acceso a los servicios de salud (Gutiérrez, 2007). Se ha seleccionado este fin de viaje dado que las personas mayores, junto a las mujeres y los niños, constituyen uno 
de los grupos poblacionales que más acuden a este tipo de servicios, incluso en forma preventiva y principalmente quienes presentan enfermedades crónicas (Piovani, 2018).

El objetivo del presente artículo es analizar la movilidad de las personas mayores desde la perspectiva de la sustentabilidad, constituye un avance de Tesis doctoral del Doctorado en Ciencias Sociales de la Universidad de Buenos Aires, que estudia las prácticas de movilidad de las personas adultas mayores y la manera en que éstas determinan el acceso a los servicios de salud.

Se trabajó con una muestra intencional, no probabilística y no representativa, conformada por 40 casos, 20 en una Institución Pública; Hospital General de Agudos ubicado en el barrio de Flores, y 20 en un servicio de la Obra Social de Jubilados y Pensionados, Unidad asistencial de Promoción, Prevención y Rehabilitación propia, en sus sedes de los barrios de San Cristóbal y Flores. Respecto de los criterios de selección de la muestra, se incluyeron personas de 60 años y más, que asisten a los Centros sanitarios seleccionados y que se encontraban en condiciones de expresar su consentimiento para responder las preguntas de la entrevista. Se excluyeron las personas que no se encontraban en condiciones psíquicas y/o cognitivas de responder y quienes no presentaron interés o voluntad de participar en el estudio. La muestra se basó en los sujetos disponibles, quienes asisten a los Centros en un día determinado, más mujeres que hombres. Teniendo en cuenta la feminización del envejecimiento no se incorporaron más casos de hombres con el fin de equiparar la muestra. La mayoría residen en la $\mathrm{CABA}$, registrándose algunas procedencias del conurbano: de la zona sur; Lanús y Avellaneda, y de la zona oeste, partido de La Matanza; Villa Celina, Ramos Mejía, Aldo Bonzi. En estos últimos casos, optan por realizar recorridos extensos, con mayor tiempo de viaje, en busca de servicios de salud que consideran de mejor calidad que los disponibles en la Provincia de Buenos Aires.

La técnica de recolección de datos consistió en un cuestionario de preguntas abiertas y cerradas administrado por la investigadora. El trabajo de campo se realizó en los meses de julio y agosto de 2018. La mayoría de las personas consultadas se ubican en la franja etárea de 60 a 79 años de edad, la de menor edad tiene 60 años y la mayor 92 años. Más de la mitad cuenta con la Obra Social de Jubilados y Pensionados, las restantes sólo cuentan con cobertura pública. Casi la totalidad de las personas son autoválidas, lúcidas, y concurren solas. Sólo 3 asistieron en silla de ruedas, con acompañante.

En el presente artículo, analizaremos parcialmente los resultados obtenidos, en el marco del nuevo paradigma de movilidad sustentable, hallazgo emergente de la investigación doctoral. La discusión sobre el tema acontece a partir del avance en las lecturas y su relación con los datos empíricos. Abordamos conceptos referidos a la sustentabilidad y las acciones implementadas en la CABA a partir de dicha meta. Luego describimos la movilidad de los hombres y mujeres entrevistados, los modos de uso y su grado de adhesión, agrupándolos en tres categorías: alto, medio y bajo o nulo. Finalmente analizamos la movilidad de las personas mayores a la luz del concepto de sustentabilidad. Los interrogantes que nos proponemos responder a lo largo de este trabajo son: ¿Cómo se movilizan las personas mayores para acceder a los centros de salud? ¿Por qué eligen usar un medio de transporte y no otro? ¿Cuáles prefieren evitar y por qué? ¿Cómo se relacionan dichas prácticas de viaje con el paradigma de la movilidad sustentable?

\section{Movilidad urbana sustentable en la CABA}

Los conceptos de sostenibilidad y sustentabilidad suelen considerarse como sinónimos, siendo imprecisos los alcances de su uso y continúan en redefinición. Surgen asociados 
al cuidado del medio ambiente y posteriormente se incorporan otras dimensiones para su valoración. La adopción de uno u otro término también fue vinculada a las tendencias ideológicas o intereses de quien lo adopta (Zarta Ávila, 2018). En los últimos tiempos, mundialmente se ha tomado conciencia de la importancia de promover una movilidad urbana sustentable, tendiendo a minimizar los impactos ambientales, energéticos y sociales derivados. Estos elementos se refieren a la reducción de emisiones, menor consumo de energía y mayor equidad en el acceso a la movilidad, respectivamente (Herce, 2009). Las formas de transporte dominante, como el automóvil particular, requieren elevado uso de combustible, genera impactos negativos en el ambiente y es restrictivo a personas de mayores ingresos. Desde esta perspectiva, el desafío es garantizar el derecho a la movilidad de la diversidad de ciudadanos sin comprometer a las generaciones futuras, evitando o minimizando los daños ambientales.

Argentina ha adherido a las metas propuestas en la Agenda 2030 para el desarrollo sostenible, aprobada en el año 2015 por la Organización de Naciones Unidas (ONU). Entre sus objetivos, se enuncia el compromiso con el cuidado del ambiente, el acceso a energías menos contaminantes y la reducción de la desigualdad, contemplando las tres dimensiones de la conceptualización que adoptamos en este trabajo.

La CABA presenta problemas similares a los de otras grandes ciudades latinoamericanas: congestión de tránsito, elevada contaminación ambiental y sonora, siniestros viales, creciente desigualdad en el acceso a derechos. En un intento de reordenamiento del servicio de transporte, a partir del año 2010 se comienzan a implementar acciones para afrontar esta problemática, desde una perspectiva de planeamiento urbano basada en la idea de modernización e incorporando una visión gerencial. Desde entonces el gobierno de la Ciudad le ha dado prioridad a las políticas de transporte, incrementando considerablemente el presupuesto destinado a esta área (Tavella, 2015). La reforma iniciada en el año 2010 comprendió el diseño e implementación de un conjunto de acciones concretas que continúan en ejecución, como la construcción del metrobus y bicisendas, la implementación del servicio de bicicletas públicas gratuitas en zonas céntricas, la restricción espacial mediante el cierre de áreas de intenso tránsito en horarios determinados de días hábiles. Estas medidas intentan agilizar los desplazamientos y restringir el uso del automóvil particular, altamente contaminante y desigual. Cabe preguntarse si se han tenido en cuenta las necesidades particulares de movilidad de grupos vulnerables y a qué población se dirigen las políticas de transporte recientemente implementadas. En la CABA, las personas mayores en general no utilizan bicicletas, los traslados a pie se ven limitados por la falta de lugares de descanso y el deterioro de veredas que obstaculizan su deambulación en el espacio público.

El lineamiento de las políticas de transporte debe apuntar a resolver las necesidades reales de movilidad de la población. Entendemos que conocer las prácticas de viaje de las personas mayores y sus significados, puede contribuir al lineamiento de políticas de transporte situadas, que apunten a la meta de la sustentabilidad sin fracasar en el intento.

En este artículo, inicialmente indagamos sobre los modos de uso y no uso, consultamos a las personas mayores sobre su forma habitual de traslado, cuál o cuáles modos no elegirían para viajar y su fundamentación. A partir de la información recopilada elaboramos una tipología de modos de uso por grado de adhesión para luego analizar las dimensiones del paradigma sustentable en los modos de mayor uso.

\section{Adhesión a la movilidad en personas mayores}

Definimos como adhesión al nivel o grado de aceptación de las personas mayores a una forma de movilidad que la convierte en un modo de uso habitual. El nivel de adhesión estará determinado por los hábitos culturales, las experiencias vivenciadas, las condiciones físicas y otros aspectos que asignan valores o disvalores a cada tipo. 
El principal modo de movilidad utilizado por las mujeres mayores consultadas es el colectivo, seguido del remis. En el caso de los hombres, se han trasladado principalmente en colectivo y a pie.

El colectivo es un bus que realiza recorridos con paradas fijas en el que las personas jubiladas y pensionadas tienen tarifas diferenciales. Hombres y mujeres mayores que se trasladan de manera autónoma lo eligen por diversos motivos: cercanía de las paradas, bajo costo, respaldo, practicidad y comodidad.

El remis, automóvil privado de alquiler, es un medio valorado muy positivamente por las personas mayores, principalmente por las mujeres, tal como señalan las siguientes entrevistadas:

"Es más seguro, los conozco de años. Es más caro pero sigo eligiendo el remis" (Jovita, 60 años).

“Es lo más confiable” (María, 74 años).

El remis permite resolver traslados en ausencia de familia u otras redes de apoyo garantizando un viaje seguro. Los motivos de no elección del remis se relacionan principalmente con su costo, considerado elevado.

Luego se consultó a las personas mayores entrevistadas, a partir del total de modos disponibles en la Ciudad, cuáles no elegirían para movilizarse y la fundamentación de la no elección. Los modos desestimados para su uso son: bicicleta, moto, tren, subterráneo, combi, Uber y traslado programado cubierto por la Obra Social. Los motivos de no elección permiten conocer posibles obstáculos o barreras identificados por las personas mayores en cada modo. El Subterráneo y Uber son los modos que no elegirían para desplazarse la mayoría de las mujeres. En el caso de los hombres, mencionan la bicicleta y Uber.

El subterráneo, tren que circula a través de un túnel y permite alcanzar alta velocidad en el desplazamiento, es un modo de uso dificultoso para las personas mayores, entre otros motivos, por la saturación de usuarios y los problemas de accesibilidad que presenta, y también por el temor que genera. Viajar en este medio en la CABA puede resultar muy complejo para personas mayores que presentan algún tipo de limitación para deambular. En horas pico, estaciones y coches colmados de usuarios, personas que transitan velozmente, tiempos muy breves para ascenso y descenso de la formación en las estaciones, escaleras mecánicas y ascensores que frecuentemente no funcionan, dificultan la accesibilidad. Así lo señalan varias personas entrevistadas:

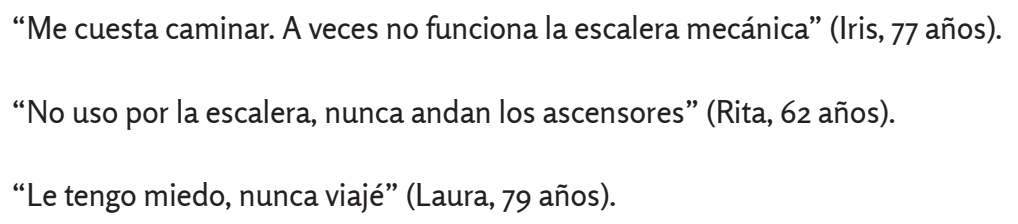

Respecto de Uber, son varios los elementos que inciden en la negativa de las personas mayores a optar por su utilización: el escaso manejo de nuevas tecnologías como celular, única forma de solicitar el servicio, y tarjetas bancarias como medio de pago. Por otra parte, la desacreditación y puesta en cuestión desde los medios de comunicación, promovida en parte por los taxistas quienes los consideran "competencia desleal", en muchos casos incide en la nula demanda de este servicio, generando prejuicios sobre el mismo. Para las personas mayores, la televisión es el principal medio de comunicación para informarse, especialmente en el grupo etáreo de 50 años y más (Hernández, 2016). 
De esta manera, presenta una fuerte presencia como formadora de opinión. Hombres y mujeres consultados argumentan sus motivos para desestimarlo:

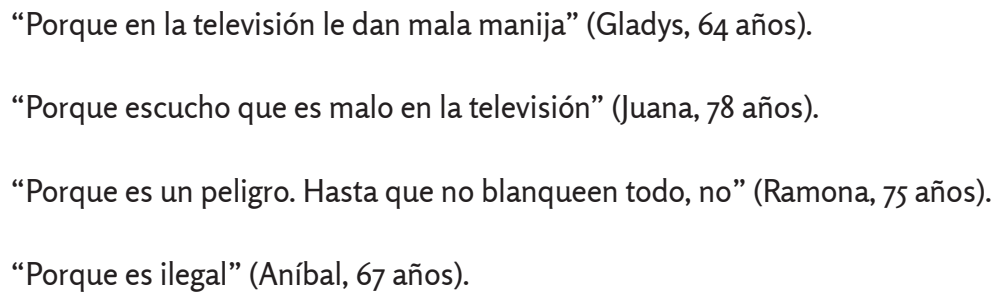

El Módulo de Traslados programados establece los servicios individuales de movilidad terrestre para afiliados a la Obra Social de Jubilados y Pensionados que presenten imposibilidad física y/o cognitiva de trasladarse por sus medios. Su finalidad es garantizar el acceso de los mismos a la prestación médica. Incluye varios servicios, como realización de estudios, pruebas y colocación de prótesis, traslados de alta, entre otros. En el caso de las personas entrevistadas en el marco del presente estudio, sólo una mujer que no deambula asistió al centro de salud mediante este tipo de traslado, acompañada por una cuidadora particular. La mayoría desconoce este tipo de prestación. Entre las personas que sí lo han utilizado, el prestigio de esta modalidad es bajo, es calificado de manera negativa por varias personas:

“Nunca vino. Tramité y no vino" (Hugo, 75 años).

“No vinieron y perdí el turno” (esposo de Estefanía, 78 años).

Este tipo de elección modal no es relevante en las personas consultadas, el desconocimiento del servicio y el procedimiento de acceso, sumado al desprestigio y las "malas experiencias", derivan en una baja adhesión de uso.

Finalmente, se indagó sobre formas alternativas en la movilidad utilizadas. Más de la mitad de las mujeres utilizan modos alternativos de traslado de acuerdo a la necesidad: subte, colectivo, tren y traslado familiar son los seleccionados más frecuentemente como segunda opción, seguido de remis y taxi. El tren, es mencionado como medio de traslado para otro fin de viaje: visitar a la familia. El colectivo es seleccionado por ser más económico, por tener tarifa diferencial y frente a la carencia de recursos para pagar los más costosos. El remis, vuelve a ser elegido por su confiabilidad, para resolver la necesidad de un traslado más protegido, para llegar a tiempo o en días con condiciones climáticas desfavorables. Esto también sucede, aunque en menor medida, con el taxi:

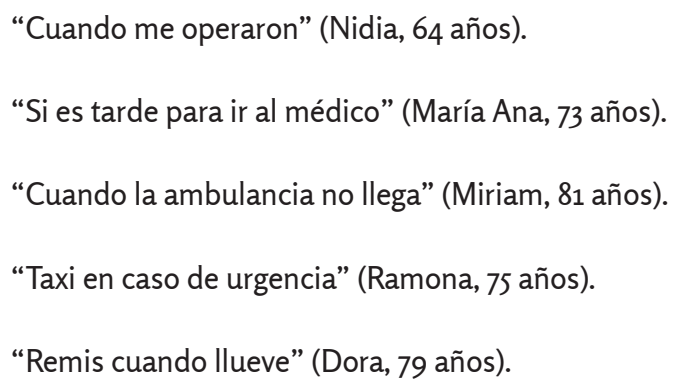

El automóvil conducido por un familiar es seleccionado por las personas mayores consultadas como medio alternativo ideal, destacando que proporciona seguridad y viaje en compañía. 
De esta manera, podemos distinguir tres categorías clasificatorias referentes a la adhesión a la movilidad de las personas mayores en los diversos modos:

» Modos de adhesión alta: Es el tipo de movilidad utilizada más frecuentemente y con mayor aceptación. Las valoraciones identificadas en esta categoría son comodidad, rapidez, accesibilidad, seguridad y tarifa diferencial en el caso de los colectivos. Estos son los modos que garantizan llegar tiempo a los servicios de salud y cumplir con el objetivo de viaje. En la muestra estudiada, integran esta categoría el traslado familiar, los colectivos y remises.

» Modos de adhesión media: Son las formas medianamente utilizadas por la coexistencia de valoraciones positivas y negativas, como la comodidad y el alto costo. El taxi y el remis, muchas veces son omitidos como modo de viaje por motivos económicos debido a su tarifa elevada.

» Modos de adhesión baja o nula: Es la oferta de movilidad no considerada por las personas mayores, por desconocimiento o por valoración negativa. Integran esta categoría las bicicletas públicas, no mencionadas por las personas mayores, ni como elegida ni como no elegida, también el Subterráneo y Uber y el traslado programado cubierto por la Obra Social.

La mitad de los hombres presentan una tendencia conservadora respecto de los modos de uso, refieren utilizar una sola forma de viaje de manera habitual. Quienes sí lo hacen, eligen tren o traslado familiar. Las personas entrevistadas, tanto hombres como mujeres, utilizan un solo tipo de transporte para trasladarse a los Centros de salud, no se observan usos combinados o multimodales. Sin embargo, observamos que es frecuente la necesidad de realizar múltiples viajes para lograr la efectiva atención: solicitud de turnos, autorizaciones y otras gestiones.

A continuación analizaremos los modos de viaje de las personas mayores en articulación con las dimensiones de la movilidad sostenible con la intención de problematizar respecto del tema y favorecer la discusión respecto de su alcance.

\section{Movilidades de personas mayores vs. sostenibilidad}

El transporte sostenible pretende “....minimizar las externalidades negativas y aprovechar las positivas, habilitando una contribución efectiva al desarrollo integral y sostenible" (Rodríguez Tornquist, 2017:189). Las externalidades pueden ser negativas, como accidentes o contaminación, o positivas, cuando facilita el apoyo al desarrollo, entre otras. Algunos modos de transporte presentan mayores externalidades negativas que otros. El uso de automóvil particular de manera individual es más contaminante, genera mayor congestión, excesivo uso de energía y es más costoso. Por su parte, los modos no motorizados, como el traslado a pie o en bicicleta, favorecen el cuidado del ambiente pero requieren de mayor habilidad física, no siempre presente en las personas mayores con algún grado de deterioro funcional y en ciudades que no lo facilitan por la carencia de infraestructura de apoyo.

La sostenibilidad incluye tres tipos combinados: la sostenibilidad energética (modos con menor consumo de energía), la sostenibilidad medioambiental (reducción de emisiones y contaminación sonora) y la sostenibilidad social, que apunta a una mayor equidad poblacional en la movilidad (Herce, 2009).

Las barreras identificadas en este estudio para el acceso a los servicios de salud, a partir de los relatos de las personas mayores consultadas, de alguna manera determinan tanto la elección modal como la cantidad de traslados. Estas barreras se relacionan 
principalmente con cuestiones organizativas, posibles de modificar. La complejidad de los procedimientos administrativos implementados en los servicios requiere de la realización de múltiples viajes para alcanzar el objetivo: solicitud personal de turnos médicos, autorización de órdenes de estudios o retirar resultados de prácticas. Otro obstáculo mencionado es la falta de comunicación de la suspensión de servicios, por ausencia del médico, paro de actividades por reclamo gremial u otros motivos. En este caso, la movilidad se torna inútil ya que no se logra el fin del viaje. El viaje inútil se define como aquel en el que no se puede concretar el servicio y fue identificado en estudios sobre movilidad y acceso a la salud de otros grupos poblacionales (Gutiérrez, 2014). También sucede que por demoras en los traslados no pueden concurrir, principalmente en el caso de los transportes programados por la Obra Social, debiendo solicitar un nuevo turno médico para su atención. Estas barreras permiten inferir que para el logro efectivo de la atención de su salud, las personas mayores deben realizar múltiples viajes, algunos evitables. Como ellos mismos señalan, se podrían generar mecanismos más ágiles para la solicitud de turnos o autorizaciones que eviten la concurrencia reiterada a los servicios. Los traslados múltiples generan mayor consumo de energía, mayor contaminación y costos económicos por tarifas. Si a ello agregamos la necesidad de mayor apoyo y modos protegidos en las personas que presentan deterioro físico o cognitivo, la externalidad negativa aumenta.

Estos conceptos nos conducen a reflexionar sobre el impacto energético, medioambiental y social de las movilidades de mayor adhesión de las personas mayores. Como se ha mencionado anteriormente, las personas que requieren de apoyo se movilizan prioritariamente en colectivo, remis y mediante el traslado realizado a través de un familiar. Para las que presentan mayor dependencia las opciones se reducen a los modos más protegidos como el remis o taxi, más costosos, con mayor impacto en todas las dimensiones del concepto de sustentabilidad.

Teniendo en cuenta el incremento sostenido de la población mayor y sus necesidades particulares de movilidad protegida, proponemos profundizar el estudio de las movilidades de las personas mayores e instalar el tema en la agenda pública para delinear posibilidades de solución a estos problemas. La simplificación de los procedimientos administrativos para el acceso a los servicios de salud es una acción factible de implementar. El uso de modos compartidos puede constituir una alternativa válida y de costo relativamente bajo. Es probable que su implementación sea dificultosa en la CABA, teniendo en cuenta la fuerte resistencia hacia los primeros colectivos y hacia el subte, que en este último caso en ocasiones persiste. En la actualidad identificamos el rechazo al servicio de Uber y la influencia de las representaciones sobre este medio en las personas de mayor edad. Otra cuestión por resolver es el bajo uso de tecnología en las personas mayores ya que estos modos requieren del uso de teléfonos móviles para la conexión. Mediante acciones concretas, basadas en las prácticas reales de movilidad de este grupo poblacional, es posible generar cambios tendientes a resolver los problemas identificados. Como señalábamos al inicio de este apartado, las estrategias de disminución de externalidades negativas pueden contribuir a la promoción de una movilidad más equitativa y sustentable en las personas mayores, que garanticen sus derechos.

\section{Conclusiones}

Las personas mayores se movilizan mediante múltiples formas de traslado y optan por modos más protegidos en caso de necesidad de apoyo, que representan un costo económico mayor. Los hábitos culturales influyen en las prácticas de movilidad de hombres y mujeres mayores. Desestiman los modos que les generan temor, como el Subterráneo, y los estigmatizados por los medios de comunicación o que requieren de 
habilidades tecnológicas, como Uber. Las condiciones económicas en muchos casos determinan el modo seleccionado para movilizarse, pero no intervienen imposibilitando el traslado en la muestra analizada.

Desde el paradigma de la Movilidad Urbana Sustentable se recomienda priorizar los modos no motorizados, a pie y en bicicleta, en los desplazamientos. Pero las personas mayores consultadas no los adoptan como forma habitual de traslado ni lo consideran como medio alternativo. Tampoco se implementa el uso de vehículos compartidos en esta Ciudad. Si consideramos las proyecciones demográficas que indican un progresivo incremento de la población mayor en los próximos años, podemos inferir un inminente problema por afrontar: más habitantes mayores, menos hábito de uso de modos no motorizados, es decir, más habitantes con menos hábito de uso de medios de movilidad sustentable, con la consecuencia de un mayor impacto ambiental, social y energético. El modo óptimo para las personas mayores, especialmente las menos móviles, es el que se realiza en vehículos: traslado familiar o remise, medios altamente contaminantes, de mayor costo y no recomendados desde el paradigma de la sustentabilidad. Por otra parte, el impacto de la contaminación afecta tanto a personas usuarias como no usuarias con lo cual el daño que genera su uso se multiplica. Estudios señalan que los vehículos de uso privado son los principales responsables del daño ambiental (Vaconcellos, 2015).

Dado que la muestra estuvo conformada en su mayoría por personas autoválidas, observamos la necesidad de profundizar el estudio incorporando a las personas más frágiles y con mayor deterioro. Podemos inferir que las personas en situación de dependencia ven restringidas sus posibilidades de uso del transporte público colectivo lo que genera la necesidad de adoptar formas más protegidas y personalizadas de movilidad, más costosas y más contaminantes.

En el diseño de políticas y con el fin de garantizar la movilidad, sería importante valorar especialmente a los grupos poblacionales de menores recursos, promoviendo modos que presenten el menor impacto posible en el ambiente, para sus habitantes y para las generaciones venideras, basados en sus necesidades particulares. Minimizar los modos más contaminantes, o utilizarlos racionalmente o en forma compartida, facilitar la deambulación a pie instalando asientos para descanso, baños públicos accesibles y centros de hidratación para personas mayores, como los históricos bebederos ubicados en las plazas, pueden contribuir en este sentido. El mobiliario urbano adecuado permite fomentar el traslado a pie en condiciones seguras, adecuadas a las personas mayores, a quienes presentan algún tipo de discapacidad y a toda la población.

La combinación de los modos de transporte disponibles, mencionada anteriormente, es una da las opciones, ya puesta en marcha en otros países. Requiere del cambio de hábitos de uso y es de bajo costo. El pasaje de remis individual o privado a compartido reduce costos, contribuye al cuidado del ambiente y procura el traslado en compañía de otras personas. La innovación estaría dada no por la generación de nuevos modos de transporte sino por la integración de modos preexistentes, teniendo en cuenta la optimización de los aspectos más valorados por las personas mayores: seguridad, confianza, costo accesible, comodidad, confiabilidad, accesibilidad.

La participación de la población en los procesos de planificación y toma de decisiones de políticas urbanas de movilidad, basadas en estudios locales, puede derivar en estrategias de mayor aceptación, hecho que no se ha evidenciado en las decisiones relativas al tema que se han adoptado en los últimos años en la CABA.

La tendencia mundial en la materia resalta la importancia de la movilidad sustentable y ecológica pero estas políticas no pueden transferirse sin partir de un diagnóstico contextual que incorpore la movilidad de sus habitantes de todas las edades, sus 
experiencias, los significados de sus prácticas. De lo contrario, pueden generar mayor exclusión en los grupos poblacionales más vulnerables, especialmente en las personas mayores más frágiles, que requieren de mayor apoyo para movilizarse. 


\section{Q Bibliografía}

» Bouchet C., Jofre M., Moyano V., Paniagua S.y Riera A. (2017). Los Ciudadanos y la Movilidad en Barcelona. Situación actual y perspectivas de futuro. España: Creafutur.

»Cataldi, M. (2018). Perspectivas de análisis para el estudio de la movilidad de las personas mayores en la Ciudad. Revista Perspectivas Metodológicas. 18 (21), 41-53. Recuperado de: www. http://revistas.unla.edu.ar/epistemologia/issue/ view/129 (06/07/2019).

»Da Cunha J. y Vignoli J. (2009). Crecimiento urbano y movilidad en América latina. Revista latinoamericana de población. Asociación Latinoamericana de población. Buenos Aires. 3 (4-5). 27-64.

" Gutiérrez, A. y Minuto D. (2007). Una aproximación metodológica al estudio de lugares con movilidad vulnerable. XIV Congreso Latinoamericano de Transporte Público y Urbano. PET-COOPE/UFR, ANPET. Rio de Janeiro. Temática Transporte y Exclusión Social, N³66. En CD ROM.

"Gutiérrez, A. (2014). Revisando el papel del transporte y la movilidad en la gestión de políticas públicas sectoriales: el caso de la salud. Programa Interdisciplinario de la UBA en Transporte (PIUBAT). Revista Transporte y Territorio. 10 (2014). 158-161.

» Herce, M. (2009). Sobre la movilidad en la ciudad. Propuestas para recuperar un derecho ciudadano. Barcelona: Reverté.

»Hernandez, A., Zovatto, D. y Fidanza, E. (comp.) (2016). Argentina: una sociedad anómica. Buenos Aires: EUDEBA.

»Instituto Nacional de Estadísticas y Censos/INDEC. (2010). Censo Nacional de Población, Hogares y Viviendas 2010. Recuperado de: www.indec.gov.ar (06/07/2019).

»Instituto Nacional de Estadísticas y Censos/INDEC (2012). Encuesta Nacional de Calidad de Vida de Adultos Mayores 2012. Serie de Estudios INDEC ${ }^{\circ} 46$. Recuperado de: www.indec.gov.ar (06/07/2019)

"Laslett, B. (1989). Gender and social reproduction: historical perspectives. Annual Review of Sociology. (15) 381-404.

» Ministerio de Desarrollo Social. Presidencia de la Nación (2010). Accesibilidad al medio físico para los adultos mayores. Argentina. Recuperado de: www.algec. org/accesibilidad (06/07/2019).

»Organización Mundial de la Salud/OMS (2007). Ciudades globales amigables con los mayores. Una guía. 2007. Recuperado de: www.who.int/ageing/ AFCSpanishfinal.pdf (06/07/2019).

»Piovani, J., Salvia, A. (Coord.) (2018). La Argentina en el Siglo XXI. Como somos, vivimos y convivimos en una sociedad desigual. Argentina: Siglo XXI Editores.

"Rodríguez Tronquist, R. y Cruz, C. (2017). Transporte sostenible. Zunino Singh D., Giucci G., Jirón P. (ed.). Términos clave para los estudios de movilidad en América Latina. Buenos Aires: Biblos.

"Sánchez González, D. (2015). Ambiente físico-social y envejecimiento de la población desde la gerontología ambiental y geografía. Implicaciones socio- 
espaciales en América Latina. Revista de Geografía Norte Grande. (6o), 97-114.

» Tavella, G. y Oniszczuk, G. (2015). La asignación de recursos y el diseño de políticas de transporte durante el gobierno del PRO. Ciencias Sociales. Revista de la Facultad de Ciencias Sociales Universidad de Buenos Aires. Buenos Aires. (87), 102-107.

"Unidad Ejecutora Central. Ministerio de Transporte de la Nación.(2010). Encuesta de Movilidad Domiciliaria 2009-2010. Recuperado de: www.uecmovilidad.com.ar (06/07/2019).

»Vasconcellos, E. (2015). Transporte Urbano y Movilidad. Reflexiones y propuestas para países en desarrollo. Buenos Aires: UNSAM Edita.

"Zarta Ávila, P. (2018). La sustentabilidad o sostenibilidad: un concepto poderoso para la humanidad. Tabula Rasa. (28), 409-423. Doi: https://doi. org/10.25058/20112742.n28.18

\section{Mariana Cataldi / marianacataldi@hotmail.com}

Licenciada en Trabajo Social (UBA) Magister en Investigación Social (UBA) Doctoranda en el Doctorado en Ciencias Sociales (UBA). Docente (UBA. Carrera de Trabajo Social), Docente e Investigadora (UNPAZ. Carrera de Trabajo Social), Docente de posgrado (Universidad Maimónides. Especialización en Gerontología Social). Publicó artículos en revistas especializadas en envejecimiento. 